\title{
EchoGéo
}

$14 \mid 2010$

Afrique, 50 ans d'indépendance : Dynamiques spatiales, identités, circulations

\section{Appliquer de nouveaux programmes scolaires : quelle géographie enseignée?}

Entretien avec Philippe Sierra, professeur

\section{Alexis Sierra}

\section{(2) OpenEdition}

\section{Journals}

\section{Édition électronique}

URL : https://journals.openedition.org/echogeo/12174

DOI : 10.4000/echogeo. 12174

ISSN : 1963-1197

\section{Éditeur}

Pôle de recherche pour l'organisation et la diffusion de l'information géographique (CNRS UMR 8586)

\section{Référence électronique}

Alexis Sierra, «Appliquer de nouveaux programmes scolaires : quelle géographie enseignée? », EchoGéo [En ligne], 14 | 2010, mis en ligne le 16 décembre 2010, consulté le 31 juillet 2021. URL http://journals.openedition.org/echogeo/12174 ; DOI : https://doi.org/10.4000/echogeo.12174

Ce document a été généré automatiquement le 31 juillet 2021.

EchoGéo est mis à disposition selon les termes de la licence Creative Commons Attribution - Pas d'Utilisation Commerciale - Pas de Modification 4.0 International (CC BY-NC-ND) 


\title{
Appliquer de nouveaux programmes scolaires : quelle géographie enseignée?
}

Entretien avec Philippe Sierra, professeur

\author{
Alexis Sierra
}

1 Philippe Sierra est docteur en géographie, professeur agrégé au lycée Raymond Naves à Toulouse.

2 - Alexis Sierra (AS). En guise d'introduction, comment percevez-vous les changements de programmes effectués en Seconde et le contexte dans lequel ils ont été réalisés?

3 - Philippe Sierra (PS). Les changements de programme ont été réalisés de manière brutale, sans concertation avec le monde enseignant qui ne s'y attendait pas et n'a été consulté que rapidement et a posteriori. Surtout, ils s'accompagnent d'une diminution horaire sans précédent avec la suppression des modules, c'est-à-dire la fin des enseignements en demi groupe dans lesquels se faisaient de nombreuses activités pratiques notamment géographiques (étude de cartes, croquis...). La disparition de l'histoire et de la géographie obligatoire en Terminale scientifique ( $51 \%$ des candidats aux bacs généraux) qui va avec la réforme des lycées, augmente encore l'inquiétude pour l'avenir de l'enseignement de nos disciplines, notamment dans les séries non générales. Ce contexte a largement brouillé la lecture du contenu des programmes de Seconde, d'autant que les inquiétudes se focalisent sur les programmes de Première.

4 - AS. Quelles sont les différences notables que vous retenez dans les nouveaux programmes de géographie?

5 - PS. On note une certaine continuité : les grands thèmes abordés dans les précédents programmes ont peu changé, à l'exception des espaces étudiés (suppression des montagnes, apparition des "espaces arctiques») et de l'introduction d'une question sur «l'enjeu énergétique ». Le fil directeur, qui est le même que celui de la classe de cinquième, donne peut-être davantage de cohérence à l'ensemble. 
6 - AS. Quelles sont les difficultés principales que vous avez perçues (ou que vous avez eues pour préparer vos nouveaux cours)?

7 - PS. Il y a une réelle difficulté à saisir la notion même de développement durable, qui est une véritable «expression valise ». Le danger est soit de la réduire à un slogan de bonne volonté, qui serait sans fondement scientifique, soit de relayer les discours simplificateurs largement médiatisés appelant à « sauver la planète ».

8 - AS. Y a-t-il eu des débats dans votre établissement, soit au moment de la consultation en ligne, soit depuis la rentrée avec la mise en application des programmes?

9 - PS. Les débats sont loin d'être clos, même s'ils concernent davantage les programmes d'histoire que les programmes de géographie, qui ont été assez bien reçus. Le fait que les épreuves de bac ne soient pas définies alors qu'elles concerneront dans à peine 18 mois plus du tiers des élèves actuellement en classe de seconde générale pose de grosses interrogations. En géographie, beaucoup de collègues reprochent le choix d'un terme trop à la mode et trop flou : le programme invite à montrer qu'il existe plusieurs modes de développement durable, mais sur quel(s) critère(s) peut-on faire reconnaître la durabilité d'un mode de développement à des élèves ? La notion de "ville durable » est également mal comprise.

10 - AS. Quelles sont les ressources que vous avez utilisées pour construire vos nouveaux cours?

11 - PS. Le fait est que les nouveaux cours doivent être conçus assez rapidement, d'autant que dans le même temps l'instauration des heures dites personnalisées a fait se multiplier les réunions chronophages nous appelant à passer de «l'individualisation » à la «personnalisation» (sic). Les manuels, qui constituent la base documentaire essentielle -notamment pour des raisons de «développement durable »- n'ont été disponibles que très tardivement et on invite -dois je dire particulièrement dans les « écolycées »?- à utiliser vidéoprojection et internet particulièrement énergivores ! J’ai fait le choix de commencer l'année en faisant lire deux extraits de texte courts mais inhabituels pour des élèves, surtout arrivant au lycée. L'un date de 1864, il s'agit un compte rendu de Reclus de l'ouvrage de G.P. Marsh - Man and Nature - et l'autre est extrait du best seller de Jared Diamond -Effondrement.

12 Pour ce qui est des ressources, avec internet et l'accès facile aux images satellites ou aux statistiques il est possible de compléter assez facilement les approches proposées par le manuel choisi, sans compter les sites bien connus des enseignants comme geoconfluences ${ }^{1}$, ou celui des cafés géographiques, sans oublier le blog désormais associé à l'émission de France culture «Planète Terre ». Les instructions mises en ligne sur eduscol ${ }^{2}$ sont pratiques pour bâtir rapidement un cours (même si la fiche qui explique le développement durable n'est pas très éclairante à mon avis !). A noter aussi que de nombreux ouvrages parus récemment sont très utiles. Pour le premier thème, je pense à celui de Y. Gunnel sur Ecologie et sociétés, chez Armand Colin, qui permet justement de mettre en perspective le regard des géographes et écologues sur la "nature", ce qui avec l'ouvrage de F. Mancebo, Le développement durable, permet d'éclairer cette notion. Pour le second, le livre de J.P. Charvet, L'alimentation dans le monde, paru chez Larousse, est très accessible à des élèves de Seconde. Pour reconstruire les cours sur la ville, je n'ai pas encore eu le temps d'y réfléchir !

13 - AS. Que pensez-vous de l'orientation générale centrée sur le développement durable? 
14 - PS. C'est une orientation «dans le vent» mais qui permet de montrer que la géographie est en phase avec le monde contemporain, et donc de maintenir l'intérêt des élèves pour les approches géographiques.

15 - AS. Comment des élèves de Seconde peuvent-ils, à votre avis, se saisir de la notion de développement durable? Comment comptez-vous vous y prendre?

16 - PS. Les élèves sont très sensibles à ces questions et de fait le débat existe vraiment. J'ai commencé par leur demander de définir sur feuille et individuellement le « développement durable ». Il a d'ailleurs été très révélateur de voir que la dimension sociale n'a été citée par aucun élève, et qu'à l'inverse les messages très concrets étaient presque systématiquement repris (trier, éteindre les lumières...). Mon objectif a ensuite été de leur rappeler que ces préoccupations ont une histoire et posent de manière pratique le regard porté sur le rapport entre les sociétés humaines et l'environnement, qui est une des préoccupations anciennes de la géographie.

17 - AS. Malgré des textes d'orientation qui précisent qu'il faut avoir une lecture complexe et systémique du développement durable, n'y a-t-il pas un risque de réduire son enseignement à une question d'écologie au sens naturaliste? Ou au contraire, est-ce une occasion de réintroduire de la géographie physique après deux décennies de mise en retrait progressive?

18 - PS. D'abord cette question sous entend que l'écologie, par laquelle est arrivée l'approche systémique, serait réductrice. Or l'écologie humaine, initiée par de nombreux géographes dès la fin du XIX ${ }^{\mathrm{e}}$ siècle, a justement ouvert des approches faisant ressortir la complexité des processus. Pour en revenir à la question, le thème n'est nullement " réduit » à une question écologique à travers les thèmes choisis, bien au contraire les instructions semblent prendre le soin d'éviter toute approche naturaliste. Il faut d'ailleurs noter que le thème 2 du programme de SVT (sol et énergie) permet en théorie des convergences souhaitées par les instructions officielles, mais peu explicitées et difficiles à mettre en œuvre (ce thème correspond à 4 ou 5 séances de TP dans lesquelles les approches géographiques ont difficilement une place et qu'à l'inverse on se voit mal utiliser en géographie!). Cette invitation des instructions a néanmoins l'avantage de souligner la complémentarité des approches. Cependant, à mon avis, les questions suscitées par les préoccupations environnementales doivent être l'occasion de réintroduire des éléments de géographie dite "physique » car les dynamiques de surface qui sont au cœur des enjeux environnementaux ne sont plus étudiées en SVT et sont totalement ignorées des élèves. Peut-on évoquer l'agriculture durable sans expliquer l'érosion des sols? Peut-on se contenter de parler du risque inondation en termes de vulnérabilité sans expliquer comment se forme une crue ? Il faut prendre garde à ne pas jargonner et à montrer que nos enseignements permettent de mieux comprendre le monde et non pas redire de manière compliquée des choses rarement ignorées de nos élèves.

19 - AS. La dernière partie du programme traite d'espaces en particulier. C'était le cas précédemment avec, au choix, les espaces littoraux et les espaces montagnards. Dans les nouveaux programmes, les enseignants n'auront plus les espaces montagnards au choix mais auront comme alternative aux espaces littoraux, les régions arctiques et les espaces soumis aux risques. Comment comprenez-vous cette dernière partie ? Quel sera votre choix? Pourquoi ? Comment pensez-vous aborder la question des espaces soumis aux risques (si c'est votre choix)? 
20 - PS. Cette dernière partie risque de passer à la trappe pour de nombreux élèves. En traitant «d'espaces en particulier» comme le dit la question, cette dernière partie permet de mobiliser une approche davantage géographique et donc de sortir d'une vision de la géographie comme science de synthèse qui fait le point sur des grands thèmes d'actualités à partir de plusieurs disciplines.

21 Elle présente une innovation intéressante en proposant parmi les leçons au choix d'étudier un espace zonal très particulier (même si les compléments au programme, très utiles et disponibles sur le site d'eduscol, invitent à rejeter une approche zonale !) les mondes arctiques- qui a l'avantage d'incarner assez facilement à la fois le "global change » et les dilemmes du développement durable. Tout le monde en a entendu parler et personne ne les connaît.

22 Le fait est que la question des « espaces soumis aux risques " pourtant si essentielle et dans laquelle les enjeux géographiques sont majeurs soit au choix et inscrite en dernière position dans le programme me semble vraiment regrettable, car voilà une leçon que malheureusement on peut bâtir chaque année sur un exemple d'actualité, qui donc intéresse particulièrement les élèves, et surtout montre l'intérêt de l'approche géographique. Je traiterai cette question, et même si elle a été reformulée et part des « espaces soumis aux risques », je l'aborderai probablement à partir d'une catastrophe.

23 - AS. Une manière de montrer comment la géographie sert à saisir un monde représenté et largement médiatisé comme incertain en somme. Nous vous souhaitons bonne chance pour atteindre vos objectifs!

\section{NOTES}

1. http://geoconfluences.ens-lsh.fr/

2. http://eduscol.education.fr/pid23208-cid52286/ressources-pour-la-classe-de-seconde.html

\section{AUTEUR}

\section{ALEXIS SIERRA}

Alexis Sierra est maître de conférences en géographie à l'université Cergy-Pontoise-IUFM. alexisierra2001@yahoo.fr 